Draft Version February 22, 2018

Preprint typeset using $\mathrm{LAT}_{\mathrm{E}} \mathrm{X}$ style emulateapj v. 5/2/11

\title{
PROBING DYNAMICS OF ELECTRON ACCELERATION WITH RADIO AND X-RAY SPECTROSCOPY, IMAGING, AND TIMING IN THE 2002 APR 11 SOLAR FLARE
}

\author{
Gregory D. Fleishman ${ }^{1,2}$, Eduard P. Kontar ${ }^{3}$, Gelu M. Nita $^{1}$, Dale E. Gary ${ }^{1}$ \\ Draft version February 22, 2018
}

\begin{abstract}
Based on detailed analysis of radio and X-ray observations of a flare on 2002 April 11 augmented by realistic 3D modeling, we have identified a radio emission component produced directly at the flare acceleration region. This acceleration region radio component has distinctly different (i) spectrum, (ii) light curves, (iii) spatial location, and, thus, (iv) physical parameters from those of the separately identified, trapped or precipitating electron components. To derive evolution of physical parameters of the radio sources we apply forward fitting of the radio spectrum time sequence with the gyrosynchrotron source function with 5 to 6 free parameters. At the stage when the contribution from the acceleration region dominates the radio spectrum, the X-ray- and radio-derived electron energy spectral indices agree well with each other. During this time the maximum energy of the accelerated electron spectrum displays a monotonic increase with time from $\sim 300 \mathrm{keV}$ to $\sim 2 \mathrm{MeV}$ over roughly one minute duration indicative of an acceleration process in the form of growth of the power-law tail; the fast electron residence time in the acceleration region is about $2-4 \mathrm{~s}$, which is much longer than the time of flight and so requires a strong diffusion mode there to inhibit free-streaming propagation. The acceleration region has a relatively strong magnetic field, $B \sim 120 \mathrm{G}$, and a low thermal density, $n_{e} \lesssim 2 \cdot 10^{9} \mathrm{~cm}^{-3}$. These acceleration region properties are consistent with a stochastic acceleration mechanism.

Subject headings: Sun: flares - acceleration of particles - turbulence - diffusion - Sun: magnetic fields - Sun: radio radiation
\end{abstract}

\section{INTRODUCTION}

Probing the acceleration region of solar flares is known to be a highly nontrivial task (Bastian et al. 2007; Xu et al. 2008; Holman 2012) since the direct emission from the acceleration site is often weaker than other competing emissions, e. g., soft X-rays (SXRs) from thermal plasma and microwave continuum from a magnetically trapped fast electron component. Recently, we identified and reported a cold, tenuous flare (Fleishman et al. 2011), which displayed neither hot coronal plasma nor a magnetically trapped population of fast electrons in a coronal loop. This rare but favorable combination of flare properties allowed us, for the first time, to firmly identify, spectrally and spatially, the radio emission component produced directly at the acceleration region and then derive physical parameters of the acceleration site and accelerated electron population.

Although detection of even a single acceleration region is important for improving our understanding of where and how the flare electrons are accelerated, it might seem discouraging because such 'clean' cases without plasma heating and electron trapping are extremely rare. In contrast, in a typical case, the flare plasma heating is an essential component, vividly seen in coronal SXR sources, while in the mi-

\footnotetext{
${ }^{1}$ Center For Solar-Terrestrial Research, New Jersey Institute of Technology, Newark, NJ 07102

${ }^{2}$ Ioffe Physico-Technical Institute, St. Petersburg 194021, Russia

${ }^{3}$ Department of Physics and Astronomy, University of Glasgow, G12 8QQ, United Kingdom
}

crowave range three source contributions (separate regions of acceleration, trapping, and precipitation, e.g., Aschwanden 1998) may be present. To study acceleration processes, it is necessary to distinguish the acceleration region contribution in the presence of these other two competing emissions. An ideal way to make this distinction, at least for cases when the acceleration is spatially displaced from the other components, is through the use of high spatial and spectral resolution microwave imaging spectroscopy, which is not yet routinely available. In the meantime, however, we are restricted to selecting rare cases where the direct emission from the acceleration region can be separated spectrally and/or temporally from the competing contribution in the spatially integrated dynamic spectrum.

Theoretical consideration of radio emission expected from the acceleration regions in flares (Li \& Fleishman 2009; Park \& Fleishman 2010) suggests that the corresponding contribution should peak at a few $\mathrm{GHz}$ and have a reasonably narrow spectrum. This is, in particular, the case of the radio spectrum from the acceleration region of the cold, tenuous flare (Fleishman et al. 2011); however, having a spectral peak at this decimeter range does not guarantee that the emission originates from the acceleration region, so some additional evidence is needed. In this paper we present the study of a flare in which a certain fraction of the radio emission (at a somewhat narrow spectral range during a limited time) can be confidently attributed to the acceleration region, even though the competing contributions are strong and overall comparable with or even dominat- 

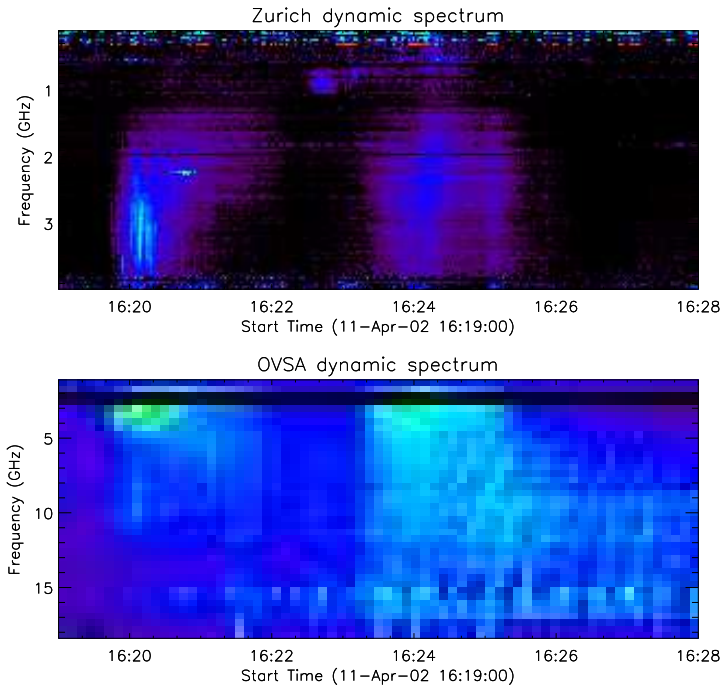

GOES10 lightcurves

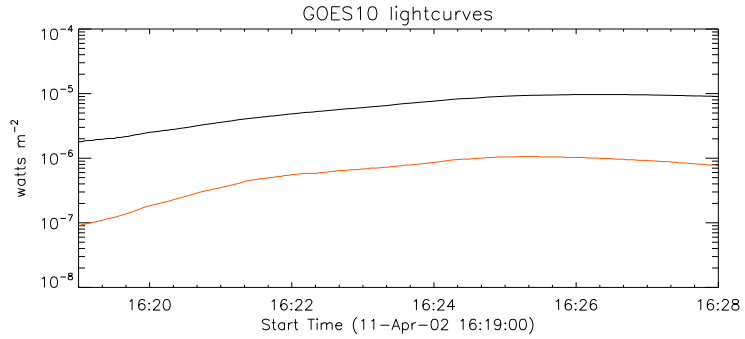

RHESSI lightcurves

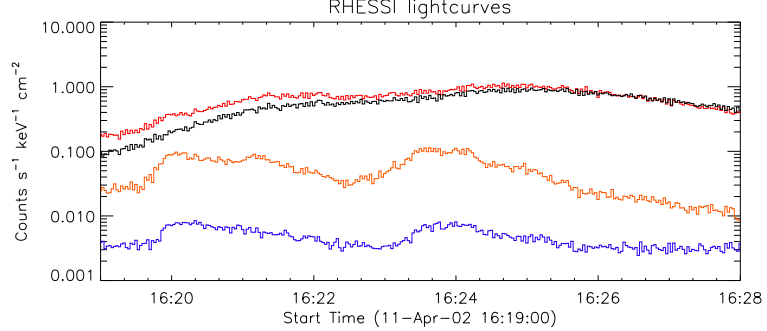

FIG. 1.- Overview of April 11, 2002 flare: Phoenix-2 and OVSA dynamic spectra, top panels. GOES (3 s) lightcurves as measured by GOES-10 spacecraft. RHESSI ( 2 second bins) lightcurves (bottom panel) in: 3-9 keV (black), 9-15 keV (red), 15-30 keV (orange), 30-100 keV (blue).

ing that from the acceleration region. In particular, we will demonstrate that the radio detection of the acceleration region in the given event is favored by stronger magnetic field at the acceleration site compared with the coronal 'trapping site', where the fast electron accumulation occurs at a later stage due to the well-known effect of magnetic trapping. Thus, the gyrosynchrotron (GS) emission from the acceleration site dominates until the number of the magnetically trapped electrons rises above the level needed to dominate over the acceleration region contribution.

In section 2 we present the observations from Reuven Ramaty High Energy Solar Spectroscopic Imager (RHESSI, Lin et al. 2002) in the X-ray range, Owens Valley Solar Array (OVSA) at 1$18 \mathrm{GHz}$, and Phoenix-2 spectrometer at $0.1-4 \mathrm{GHz}$ (Messmer et al. 1999). Context SXR observations made by GOES-10 and SOHO/MDI measurements of the photospheric magnetic field are utilized as well along with a new 3D modeling tool, GX_Simulator,
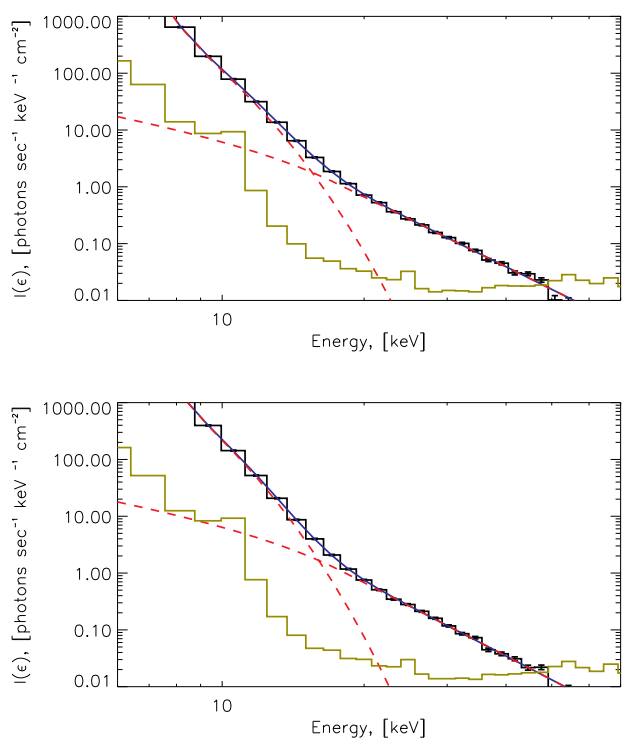

FIG. 2.- RHESSI spatially integrated X-ray spectrum above $6 \mathrm{keV}$ for two HXR peaks of the event: $16: 19: 43-16$ : 22 : 01 UT - top panel and 16:23:07 - 16: 25:13 UT lower panel. In both panels, brown line indicates background, red-dashed lines thermal and non-thermal components, blueline is the total fit (thermal plus non-thermal), and black and brown histograms X-ray data for the signal and the background respectively.

which we have developed and recently included into Solar Software 4 . In section 3 we present spectral fitting of the radio and hard X-ray spectra using 5 to 6 physical parameters, and show that the acceleration region can be separately identified. We outline a possible 3D geometry in section 4 and conclude with a discussion of the results in terms of a stochastic acceleration mechanism.

\section{OBSERVATIONS}

\subsection{X-ray Imaging and Spectroscopy}

The flare demonstrates two hard X-ray peaks as evident from Figure 1. The spatially integrated RHESSI X-ray spectrum (Figure 2) over the duration of each peak indicates the presence of both thermal and nonthermal components as often seen in X-ray spectra (e.g. see Kontar et al. 2011, as a review). To achieve better count rate statistics and energy resolution, we summed all front segment detectors but the detectors 2 and 7 were avoided (see Smith et al. 2002 for the details). Spectral analysis was done using OSPEX (Schwartz et al. 2002) with systematic errors set to $0.02 \%$. As the flare appeared on the solar disk $\left(x \simeq 500^{\prime \prime}, y \simeq-180^{\prime \prime} ;\right.$ heliocentric angle $\simeq 56^{\circ}$ ), albedo correction (Kontar et al. 2006) was applied assuming isotropic emission (minimum correction). The spectrum was fitted with a standard thermal plus non-thermal thick-target model (Figure 2), with the isotropic bremsstrahlung cross-section following Haug (1997).

The temporal variation of plasma parameters obtained from the HXR fit with time is presented in Figure 3. The derived accelerated electron spectral

\footnotetext{
4 Www.lmsal.com/solarsoft/ssw_packages_info.html
} 


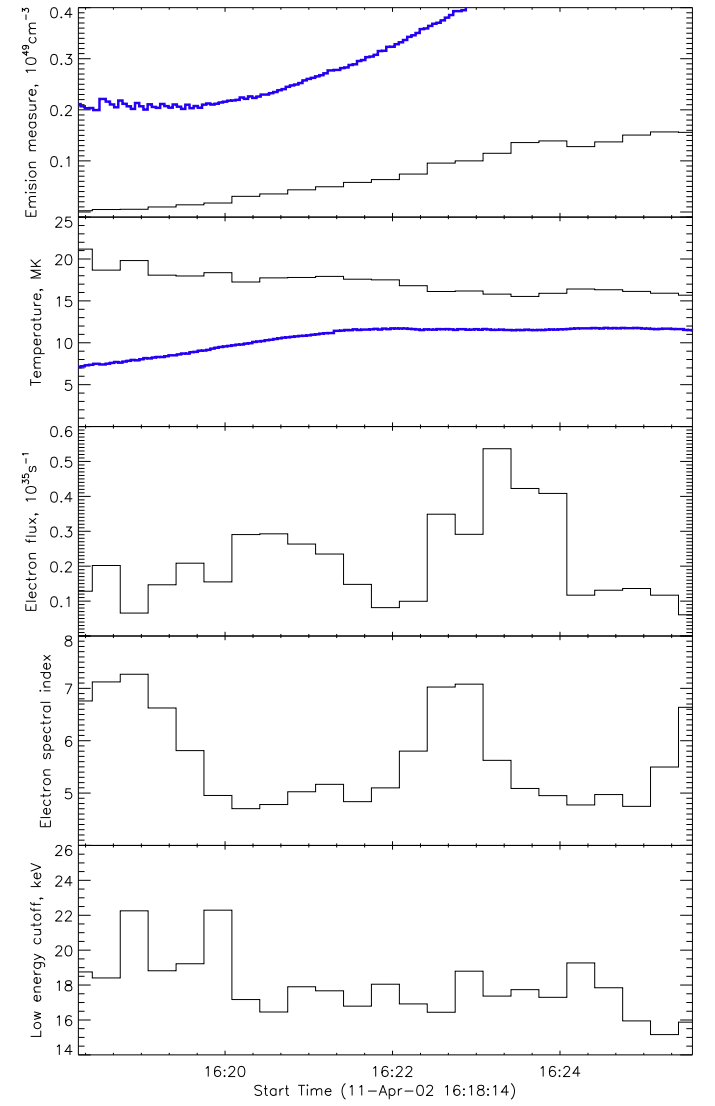

FIG. 3.- Spectral fit parameters from the thermal plus thicktarget X-ray model for 20 second time intervals of RHESSI data (black curves) as well as emission measure and temperature as obtained from GOES-10 data (blue curves).

evolution demonstrates typical soft-hard-soft behavior and shows two clear peaks in hard X-rays, well visible in $30-100 \mathrm{keV}$ lightcurves (Figure 1). The emission measure of the thermal plasma increases from $\lesssim 10^{47} \mathrm{~cm}^{-3}$ to $\sim 10^{48} \mathrm{~cm}^{-3}$, while the temperature of the plasma stays rather constant, ranging from $\sim 20$ to $\sim 15 \mathrm{MK}$ over the course of the flare. The emission measure and the temperature observed by GOES-10 are somewhat higher and lower, in the ranges above $2 \cdot 10^{48} \mathrm{~cm}^{-3}$ and between 12 and $10 \mathrm{MK}$, respectively, but show a similar temporal behavior.

RHESSI imaging (Hurford et al. 2002) reveals a spatially well-defined soft X-ray (6-12 keV and 12-20 $\mathrm{keV}$ ) source (Figure 4), visible over the entire time of the flare, whose location gradually moves eastward by roughly $10^{\prime \prime}$ over the flare duration. HXR sources imaged in the $20-40 \mathrm{keV}$ range have higher temporal variability, which coincides spatially with the SXR source at the troughs of X-ray light curves at 16 : $19 \mathrm{UT}$ and $16: 23 \mathrm{UT}$ but deviates from this location at the peaks, when the electron acceleration rate is the highest. Based on SXR-toHXR spatial relationship alone, one could interpret the event in terms of the standard flare picture, i.e. a looptop SXR source and two uneven HXR footpoints. This interpretation, however, is supported neither by the magnetic field data shown in Figure 5 nor by the observation-based 3D modeling given below in Fig. 10. We argue based on analysis of
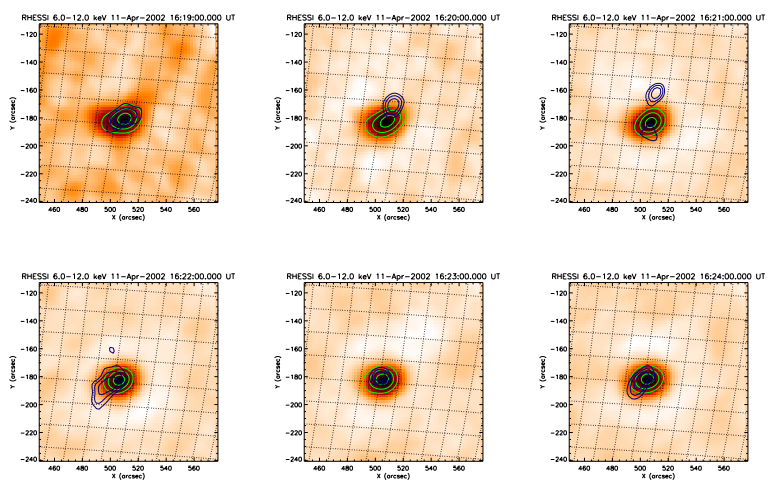

FIG. 4.- Spatial distribution of X-ray emission from April 11, 2002 flare in various energy ranges. Background image $6-$ $12 \mathrm{keV}, 12-20 \mathrm{keV}$ green (contours are at 50, 70, $90 \%$ levels), and $20-40 \mathrm{keV}$ dark blue (contours are at 70, 80, $90 \%$ levels). The X-ray images are reconstructed using clean algorithm.

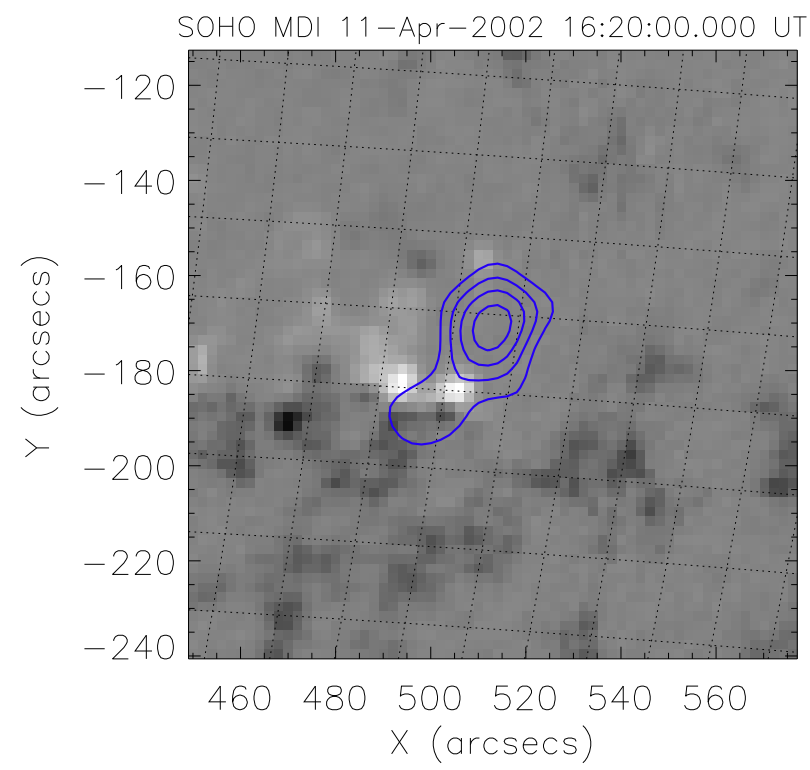

FIG. 5. - 20-40 keV HXR contours [45; 60; 75; 90\%] for 16:20-16:21 UT superimposed on the SOHO/MDI line of sight magnetic map. It is clearly seen that the HXR source is projected to the photosphere region free from any noticeable magnetic field enhancement, which supports the idea that the HXR source is coronal, rather than a footpoint.

the microwave data, which we will show later, that the main HXR source remains coronal most of the time, but support for this can be seen in the fact that the 20-40 keV source in Fig. 5] does not overlie any concentration of magnetic field, that could correspond to footpoints often seen in flares (e.g. Kosugi et al. 1992; Emslie et al. 2003; Kontar et al. 2010; Battaglia \& Kontar 2011). The only time period when the main HXR source possibly could be identified with a footpoint is late in the first peak 16:21- 16:22 UT when the $20-40 \mathrm{keV}$ source is more strongly displaced to the north from the SXR source, while later the HXR source moves back slightly to the south. A weaker HXR source, seen as a south-east extension of the main HXR source in Figures 5 and 6 . left, could correspond to a footpoint; alternatively, it can correspond to another part of the same magnetic 

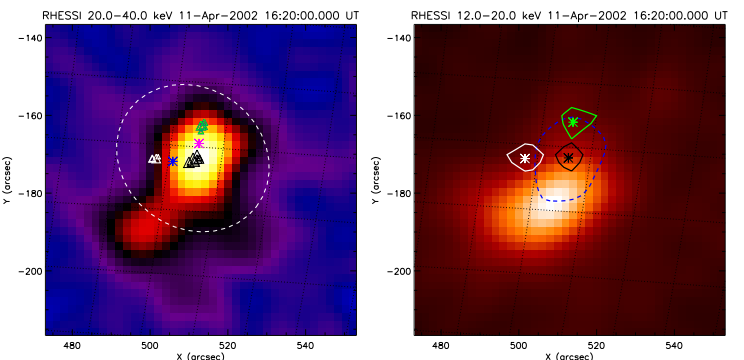

FIG. 6.- Left: Evolution of the spatial brightness peak of the radio emission at $2.6-4.2 \mathrm{GHz}$ from April 11, 2002 flare. Background: HXR image at $20-40 \mathrm{keV}$ at 16:20:0016:21:00 UT. Symbols are OVSA image centroid positions separated by $8 \mathrm{~s}$ time interval (OVSA temporal resolution for imaging): green triangles are for snapshots from 16:19:55 to 16:20:15 UT, pink asterisk for 16:20:20 UT, black triangles from 16:20:25 to 16:22:00 UT, white triangles from 16:23:40 to $16: 24: 20 \mathrm{UT}$, and the blue asterisk is for a late decay phase of 16:25:08 UT; larger triangles correspond to later snapshots within each group. The sequence of the contours clearly indicates that the radio source is located at the northern part of the HXR image and stays there during the entire impulsive phase, then moves southward to exactly match the HXR centroid position and stays there the entire decay phase of the first peak, then it moves westward during the second peak, and finally returns back to HXR centroid location at the late decay phase. The accuracy with which the contours with the same color coincide with each other indicates the accuracy of the position measurements. The synthesized bean is shown by the dashed white oval. Right: centroids and 95\% contours of the three dominant source (corresponding to the green, black, and white sources on the left panel) positions superimposed on the SXR image; blue dashed contour shows the HXR source at $60 \%$ level.

structure or to a lower, more compact loop.

\subsection{OVSA imaging}

We employ OVSA frequency synthesis imaging around the spectral peak frequency, 2.6-4.2 GHz, while no reliable imaging was possible at the higher frequencies, $\sim 10 \mathrm{GHz}$ due to the low flux levels. Given a limited OVSA spatial resolution at low frequencies, the radio sources were unresolved, although useful information on the source location at different stages of the burst has been obtained.

Figure 6 displays the centroid location of the radio emission during different stages of the event superimposed on the HXR and SXR images plotted for time interval 16:20:00-16:21:00 UT. A few important conclusions can be made based on the presented spatial relationships. First, we note a clear spatial evolution of the radio source, which is initially (during the impulsive peak of low-frequency microwave emission at $2.6-4.2 \mathrm{GHz}$ ) displaced by roughly $10^{\prime \prime}$ from the accompanying HXR source with even greater displacement, by roughly $20^{\prime \prime}$, from the accompanying SXR source. Upon transition to the decay phase, the radio brightness peak shifts by $\sim 10^{\prime \prime}$ to exactly match the brightness center of the HXR source, which remains displaced $10^{\prime \prime}$ from the SXR source. Finally, during the second temporal peak of the flare around 16:24 UT, the radio brightness peak is displaced by $10^{\prime \prime}$ to the east, after which it eventually returns back to the location of the radio source during the decay phase of the first flare peak.

\subsection{Radio to X-ray Timing}
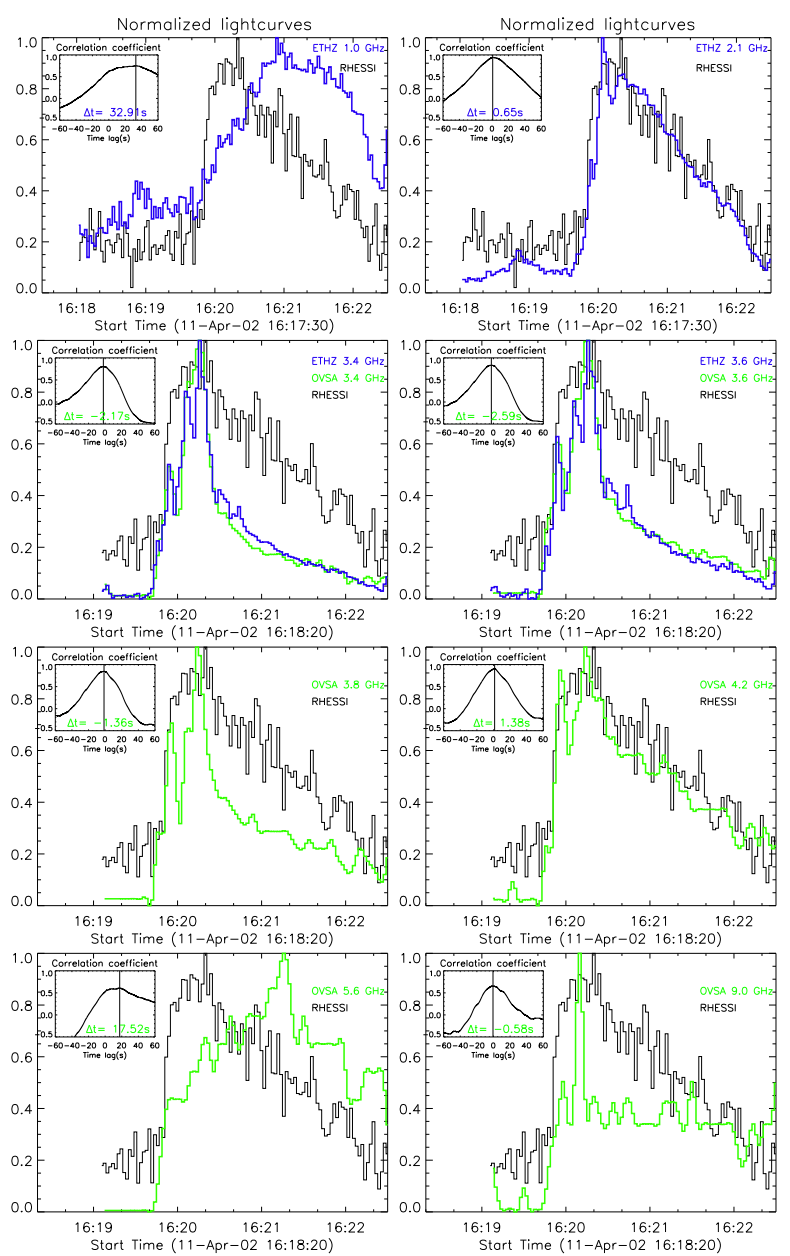

Fig. 7.- Radio to HXR timing with Phoenix-2 and OVSA at different frequencies. Black thin curve, same throughout all the panels: HXR (20-40 keV) light curve with 2 s resolution normalized to 1; blue thick curves: resampled (to the RHESSI $2 \mathrm{~s}$ resolution) Phoenix-2 light curves at different frequencies indicated at the panels; green curves: spline interpolated OVSA light curves from original $4 \mathrm{~s}$ resolution to the RHESSI $2 \mathrm{~s}$ resolution at different frequencies indicated at the panels. The insets: corresponding Radio-to-HXR lag correlations in which the time delay is printed by either blue (for Phoenix-2 data) or green (for OVSA data). The light curves at other frequencies are given in Figs. 11 15 in the end of the file.

Study of the emission light curves including crosscorrelations and delays between various microwave and X-ray channels is often highly helpful in identifying distinct emission components, which as noted in the introduction may be produced by electrons at the acceleration site, by magnetically trapped electrons, or by precipitating electrons. Figure 7 displays temporal relationships between radio light curves recorded by Phoenix-2 and OVSA on one hand and HXR light curves recorded by RHESSI on the other hand. To perform the lag cross-correlation analysis between the radio and HXR light curves, the Phoenix-2 light curves were resampled, while OVSA light curves spline interpolated to match $2 \mathrm{~s}$ RHESSI resolution. The displayed radio light curves are distinctly different from each other depending on the frequency and can be grouped as follows.

The low frequency radio light curves, $f<2 \mathrm{GHz}$, display a significant time delay compared with the 
HXR light curve: up to half a minute as determined from lag cross-correlation and up to $45 \mathrm{~s}$ if the peak times are considered. Then, at higher frequencies, $2 \mathrm{GHz}<f<5 \mathrm{GHz}$, there is no significant delay between the light curves; the delay varies within $\pm 2 \mathrm{~s}$ in this frequency range. However, the various radio light curves do show spectral evolution within this range: they become more and more impulsive as frequency changes from 2 to $2.8 \mathrm{GHz}$, then the light curves stay roughly similar to each other at $2.8-3.8 \mathrm{GHz}$, and then they again become less impulsive at $4-5 \mathrm{GHz}$. At higher frequencies, $5.0-7.5 \mathrm{GHz}$, the radio light curves are again significantly delayed compared with the HXR light curve similarly to the lowest frequency range. Finally, the highest frequency range is characterized by light curves with an impulsive peak coinciding with the HXR peak and with the impulsive radio peak at frequencies $2.8-3.8 \mathrm{GHz}$, but followed by a more extended part of the light curves.

We conclude that the radio light curves consist of two essentially different components -impulsive and delayed - whose relative contributions depend strongly on the frequency The delayed radio light curves behave exactly as expected for the microwave emission produced by a magnetically trapped component of fast electrons (e.g., Melnikov 1994; Bastian et al. 1998; Kundu et al. 2001; Melnikov 2006). In contrast, the impulsive radio light curves around the spectral peak frequency of $3.2 \mathrm{GHz}$ reach maximum flux simultaneously with the HXR light curve and so cannot be ascribed to the trapped component. Instead, their timing shows remarkable similarity to that found in the cold, tenuous flare (Fleishman et al. 2011), i.e. microwave emission produced directly from an acceleration region at low frequencies and from precipitating electrons at high frequencies. We suggest that the impulsive and delayed emissions come from physically different sources, which is consistent with their different spatial locations discussed in the previous section.

\section{RADIO SPECTRAL FIT}

An alternative way of looking at spectral evolution, rather than frequency-dependent light curves, is through time-dependent spectra during the burst. These are shown in Figure 8 which includes both the measured total power radio spectra (points) and spectral fits to be described below. The observed spectral evolution is in marked contrast with the event of 2002 July 30 analyzed by Fleishman et al. (2011), where almost no spectral evolution was present. Indeed, Fleishman et al. (2011) successfully fitted the entire sequence of the radio spectra in the 2002 July 30 event with a source model having only one free parameter - the instantaneous number of the accelerated electrons at the source. Judging from the significant spectral evolution in the event under study, such a simplified approach to the radio spectral fit cannot work. A multi-parameter fit similar to that developed by Fleishman et al. (2009) is needed in

\footnotetext{
${ }^{5}$ No impulsive component (acceleration region contribution) is detected during the second peak of the flare occurring around 16:24:00 UT; not shown in Figure 7
}

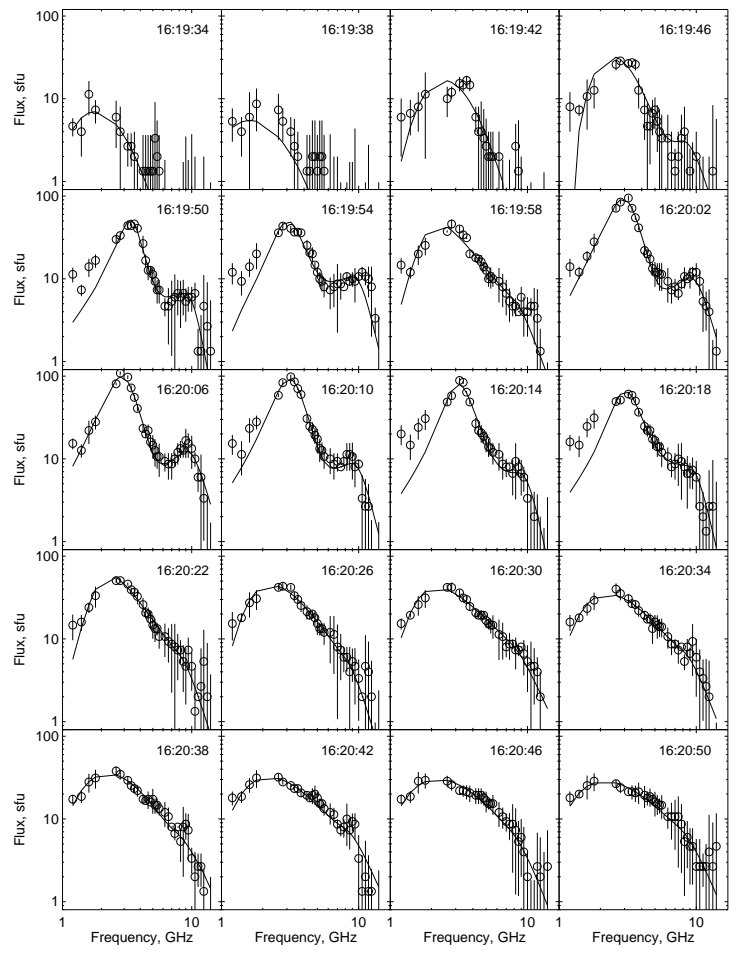

FIG. 8. - Radio spectral fit as described in the text (solid curves) of the OVSA spectra (symbols with scatter) at consequent snapshots with $4 \mathrm{~s}$ cadence; Part I. Other spectra with fits are given in Figs. 16 19 below.

this case. However, Fleishman et al. (2009) applied their multi-parameter fit to a sequence of spatially resolved (model) spectra, while here we are forced to deal with the total power spectrum, in which source nonuniformity can be expected to play a major role (Kuznetsov et al. 2011).

Note that many of the spectra in Figure 8 show two spectral peaks. A similar two-component structure was present in all radio spectra in the 2002 July 30 event (Fleishman et al. 2011), which was successfully interpreted as a combination of a main (lowfrequency) coronal source, representing in fact the acceleration region in that event, and a secondary (higher-frequency) source formed by the precipitating component of the fast electrons. Here we employ a similar two-component spectral model, i.e., that the radio spectrum is formed in two uniform sources physically related to each other. While many of the radio spectra do not show two clearly separated spectral peaks, nevertheless, we fit two homogeneous spectral components at all times because they are needed to account for the source inhomogeneity, which broadens the spectrum to the extent that it cannot be fitted by a single-component, homogeneous GS spectrum.

Specifically, we adopt the premise that the bulk of low-frequency emission comes from a coronal source with unknown (free) parameters such as magnetic field $B_{1}$ and thermal density $n_{e}$, where a power-law population of fast electrons is present with an unknown number density $n_{r}$, spectral index $\delta$, and highenergy cut-off $E_{\max }$, but with a fixed low-energy cutoff $E_{\min }=17 \mathrm{keV}$, as shown in the lower panel of Figure 3. The GS emission, especially its polarization, 
depends on the viewing angle and angular distribution of the fast electrons (e.g., Fleishman \& Melnikov 2003). Since no polarization data are available, we fix the viewing angle at a quasi transverse value, $\theta=80^{\circ}$ and adopt the simplest case of an isotropic electron distribution.

Based on the imaging data we fix the main source size to be $25^{\prime \prime}$ in each dimension so the low-frequency source is fully characterized by these five free parameters. For the second ('precipitating') source we begin with one free parameter, the magnetic field $B_{2}$, keeping $n_{e}, E_{\min }$, and $E_{\max }$ values the same as for the 'coronal' source. The spectral index of the instantaneous distribution of precipitating electrons is taken to differ by $1 / 2$ from that of the main electron population, $\delta_{p r}=\delta+1 / 2$, to account for the time of flight effect, $\tau_{\mathrm{TOF}} \sim L / v \propto E^{-1 / 2}$, which assumes energyindependent escape and a free propagation transport regime for the precipitating electrons, and the size of the high-frequency source is corrected to conserve magnetic flux along the flaring magnetic flux tube. Certainly the adopted precipitation model may be oversimplified (see, e.g. van den Oord 1990; Su et al. 2011; Holman 2012) but it is sufficient for the spectral fitting and allows clear isolation of the low-frequency spectral component during the time when the double peak structure is clearly present in the radio spectrum. Since our focus is on the low-frequency component, we forego more-detailed discussion of the precipitating source. The gyrosynchrotron source function is computed by the fast GS code developed by Fleishman \& Kuznetsov (2010).

Although this six-parameter fitting succeeds throughout the entire flare duration, there is a mismatch between the fit and observed spectrum at some time frames at the lowest frequencies. This is a wellknown indication of source nonuniformity (Nita et al. 2004; Altyntsev et al. 2008; Qiu et al. 2009), which could have been accounted by adding a contribution from the electron accumulation site (see below); we avoid this complication, however, because the parameters of this third spectral component could not be reliably constrained from the fit. The derived physical parameters show noticeable scatter, particularly in the magnetic field $B_{2}$, whose values clustered around several discrete levels near 800,400 , and $200 \mathrm{G}$ during different flare stages 6 . Accordingly, to improve stability of other derived parameters we fixed the $B_{2}$ (whose exact value is inessential to what is discussed below, but needed to obtain a good fit, especially, for the spectra with two peaks) to one of the above values based on the original six-parameter fit, for time ranges corresponding to peak 1 impulsive phase $(800 \mathrm{G})$, peak 1 decay phase $(400 \mathrm{G})$, peak 2 rise phase $(200 \mathrm{G})$ and peak 2 decay phase (again $800 \mathrm{G})$, as is explicitly shown in Figure 9 We then repeated the fitting for the remaining five free parameters in the low-frequency ('coronal') source, and the same parameters with the above-stated adjustments in $\delta$ and source size for the high-frequency ('precipi-

6 In fact, when $B_{2} \simeq 200 \mathrm{G}$, the contribution from this source is negligible, thus, a one-component GS fit works well during this period of time.
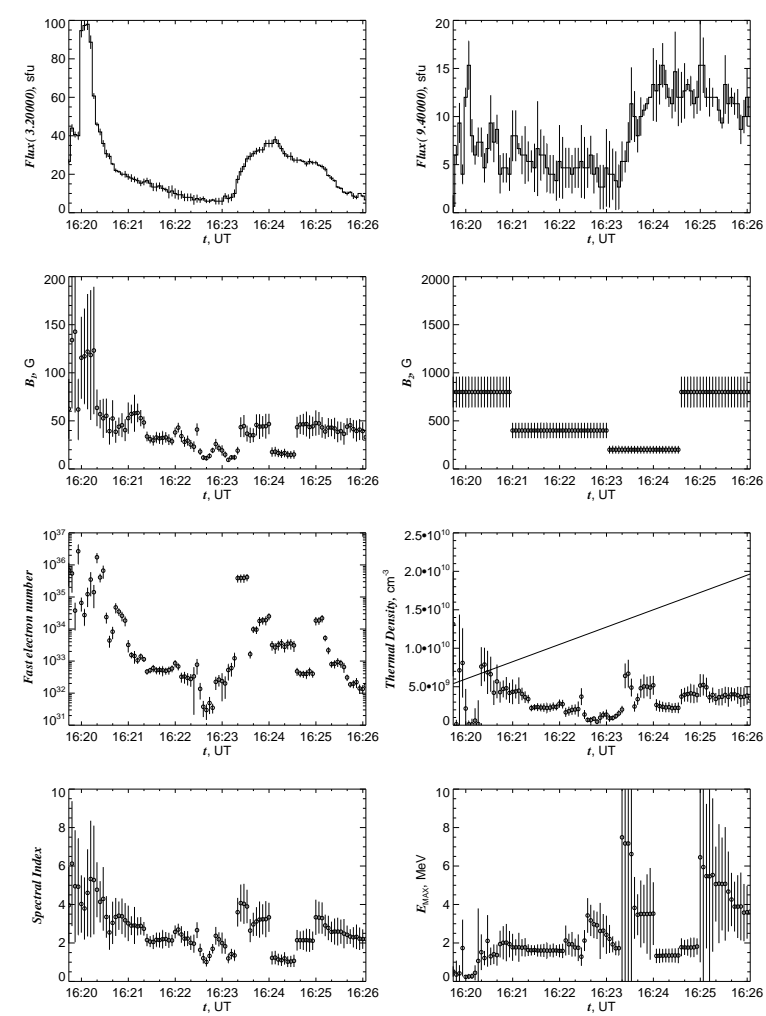

FIG. 9.- Radio source parameters as derived from the OVSA spectral fit for five parameters of the low-frequency coronal source and adopted magnetic field value $B_{2}$ for the 'precipitating' source as described in the text. A solid curve at the thermal plasma number density shows a number density evolution of the SXR source derived from emission measure from the RHESSI fit. Two top panels show the radio light curves recorded at $3.2 \mathrm{GHz}$ and $9.4 \mathrm{GHz}$ given for the reference purpose.

tation') source, therefore, the presence of the precipitation source does not add any additional free parameter to the model. The time evolution of the derived parameters of the coronal source and adopted magnetic field $B_{2}$ of the precipitation source is shown in the bottom six panels of Figure 9, compared with the observed 3.2 and $9.4 \mathrm{GHz}$ microwave time profiles (upper panels).

The derived evolution of the physical parameters deserves some discussion. The magnetic field in the low-frequency source is about $120 \mathrm{G}$ during the impulsive phase of the radio burst, while it drops quickly to 30-50 G at the transition to the decay phase around 16:20:20 UT. Remarkably, this magnetic field change derived from the spectral fit, Figure 9, happens at the very same time as the $10^{\prime \prime}$ shift of the spatial brightness peak, see Figure 6. This implies that it makes sense to distinguish between these two spatially distinct low-frequency sources, which as we show below represent the very acceleration region (the early source, with $B \sim 120 \mathrm{G}$, producing the impulsive radio emission) and the classical looptop radio source (the later source, with $B \sim 40 \mathrm{G}$, producing the radio emission from magnetically trapped electrons over the decay phase) spatially coinciding with the HXR source.

Acceleration region. At the impulsive lowfrequency source, $\sim 16: 20: 00-16: 20: 20 \mathrm{UT}$, the ther- 
mal number density obtained from the radio fit is somewhat low, $n_{e} \lesssim 2 \cdot 10^{9} \mathrm{~cm}^{-3}$, implying the radio source is located in the corona, not at a chromospheric footpoint, while the number of nonthermal electrons is consistent with the acceleration rate derived from the HXR data, $(1-3) \cdot 10^{34}$ electron/s, see Figure 3. if they reside at the radio source for 2-4 s, which requires the strong diffusion transport mode. The radio derived electron spectral index does not display any significant departure from the HXR derived electron spectral index during this time interval, cf. Figure 3. All these properties are similar to those determined for the acceleration site in the 2002 July 30 event (Fleishman et al. 2011), from which we conclude that we have here another instance of the acceleration region detection in a solar flare. The electrons accelerated at this source escape from there in roughly $3 \mathrm{~s}$ and then accumulate in another, 'trapping' source, which dominates the radio spectrum and spatial location after 16:20:20 UT. The acceleration, however, continues for a longer time: we note that the maximum electron energy, $E_{\max }$, displays a monotonic increase from $\sim 300 \mathrm{keV}$ to $\sim 2 \mathrm{MeV}$ over this phase of the burst ( 16:20:00-16:20:50 UT), which is reasonable to interpret as the growing of a powerlaw 'tail', i.e., the very process of the electron acceleration. Thus, the impulsive low-frequency source dominating the radio emission over roughly 16:20:0016:20:20 UT, which produces fast electrons and supplies them to the coronal trapping site until at least 16:20:50 UT, can confidently be identified with the acceleration region of the flare under study.

Electron accumulation site. Transition to the gradual decay phase 7 at about 16:20:20 UT manifests the stage when the trapping site has accumulated a sufficient number of fast electrons to dominate the radio spectrum. At this time the derived number of accelerated electrons with $E \gtrsim 20 \mathrm{keV}$ reaches a maximum of $\sim 10^{36}$ electrons, which corresponds to the number density of the accelerated electrons of $n_{r} \sim 2 \cdot 10^{8} \mathrm{~cm}^{-3}$ for the adopted source volume. For the determined earlier acceleration rate of $(1-3) \cdot 10^{34}$ electron/s, having a total electron number of $\sim 10^{36}$ requires a highly efficient electron trapping with the trapping time longer than $30 \mathrm{~s}$. Indeed, that long trapping time is fully confirmed by the measured delay between the HXR/impulsive radio light curves and 'non-impulsive' radio light curves, see Figure 11, some of which are delayed by almost one minute. This means that in this electron accumulation site the low-frequency radio emission is dominated by the magnetically trapped electron component, which is often seen in microwave bursts (e.g. Melnikov 1994; Melnikov \& Magun 1998; Bastian et al. 1998; Lee \& Gary 2000; Kundu et al. 2001; Melnikov et al. 2002; Bastian 2006; Tzatzakis et al. 2008; Reznikova et al. 2009). In contrast to the strong diffusion mode in the acceleration region established above, effective mag-

\footnotetext{
${ }^{7}$ Its main parameters, $B_{1} \sim 30-50 \mathrm{G}$ and $n_{e} \lesssim 5 \cdot 10^{9} \mathrm{~cm}^{-3}$, clearly indicate its coronal location, although spatially distinct from the acceleration region showing a different coronal location.
}

netic trapping implies a weak diffusion mode at the accumulation site, mediated, perhaps, by Coulomb collisions.

Indeed, it is well known (e.g., Melrose \& Brown 1976; Melnikov 1994; Bastian et al. 1998; Melnikov \& Magun 1998; Lee \& Gary 2000; Melnikov 2006) that Coulomb losses of the trapped electrons result in a flattening of the fast electron energy spectrum. This effect must be present and accounted for since the Coulomb loss time is about $10 \mathrm{~s}$ for $20 \mathrm{keV}$ electrons, which is much shorter that the trapping time at the decay phase of the burst. Indeed, the recovered evolution of the electron spectral index (Figure 96) displays a monotonic decrease over the entire decay phase, nicely in agreement with the theoretical expectation, while the maximum electron energy remains roughly constant at the level of $\sim 2 \mathrm{MeV}$ implying that no acceleration to even higher energy is demanded by the radio spectrum fit. Although some of the details of the accumulation site interpretation are not unambiguously confirmed, e.g., some regimes of the wave scattering can result in a weak diffusion mode, (see, e.g., Bespalov et al. 1987; Stepanov \& Tsap 2002), and still be compatible with efficient magnetic trapping, our main point is that this radio source (coinciding with the HXR source) is distinctly different from the acceleration region source, discussed above.

In addition to the spectral index evolution, the fit results also suggest a modest decrease of the magnetic field and the thermal number density at the radio source. This behavior can be understood if we suppose, as is likely, that the flaring loop is nonuniform with height in such a way that higher field lines are linked with more tenuous thermal plasma (implying slower Coulomb loss rate) than more compact lower field lines. In this case the trapped fast electrons will live longer at the outer field lines, so the outer looptop regions (with lower magnetic field and smaller thermal density) will dominate the radio emission at the late decay phase. We note that this source remains at the same projected position during the entire decay phase as seen from the OVSA imaging. The derived thermal number density in the radio source is always below the thermal number density derived from the emission measure determined from the RHESSI Xray spectral fit, which is consistent with significant spatial displacement between these two sources.

The described fit results are highly stable vs. various modifications of the fitting process including varying weights of the data points and initial values of the fitting parameters used as input. In contrast, the fit is not so stable during the second flare peak around 16:24 UT, which is a direct consequence of broader observed radio spectra, indicative, perhaps, of a stronger role of source inhomogeneity at this second peak making the spectral fit not unique. Nevertheless, the overall range of the derived parameters is consistent with that for the first flare peak, although no direct contribution from the acceleration region is detectable here.

\section{DISCUSSION AND CONCLUSIONS}


We have demonstrated that the combination of $\mathrm{X}$ ray and microwave imaging, spectroscopy, and timing may allow a firm detection of the solar flare acceleration site even when the thermal SXR emission and microwave contribution from a magnetically trapped electron population are strong. For the 2002 April 11 event discussed here, the direct radio emission from the acceleration region dominates the radio spectrum during the early, impulsive phase of the radio emission at $2.6-4.2 \mathrm{GHz}$.

For a better understanding of the acceleration region and its relationship with other emission components it would be highly desirable to somehow constrain the 3D geometry and magnetic connectivity within the flaring region. The current state-of-the-art of magnetic field reconstruction implies using a nonlinear force-free field (NLFFF) extrapolation of vector magnetic field measurements at the photospheric level to the corona. In our case, however, no vector magnetogram is available, but the line-of-sight data only from SOHO/MDI. This makes the 3D modeling described below non-unique, and is only offered for illustrative purposes.

To build a 3D model we use our open 8 modeling tool, GX_Simulator, and start from the line-of-sight $\mathrm{SOHO} / \mathrm{MDI}$ photospheric magnetic measurements at the region of interest. We then have the choice to apply either potential or linear (constant- $\alpha$ ) force-free field (LFFF) extrapolations to build a 3D magnetic data cube with which we can plot the field lines and form flux tube around some of them. Our tests with different values of force-free parameter $\alpha$ show that it is not possible to build a magnetic structure consistent with locations of the $\mathrm{X}$-ray and radio sources for either the potential field $(\alpha=0)$ or for most LFFF extrapolations. However, we succeeded to find the required connectivity for $\alpha \approx-5.5 \cdot 10^{-10} \mathrm{~cm}^{-1}$. We use the corresponding magnetic data cube for our $3 \mathrm{D}$ illustrative model, summarized in Figure 10. The left column of panels shows, respectively, the perspective, side, and top views of the magnetic structure in the flaring region. The locations of the acceleration region (star symbol) and trapping source (circle) are shown in relation to this structure.

It is interesting that the trapping source matches perfectly the top of the flaring loop as expected, while the acceleration region source is displaced compared with the loop top toward a region where many of the magnetic field lines are open. Moreover, inspection of neighboring field lines implies that formation of a cusp nearby to the acceleration region is likely. Speculating further, one might conclude that the particle acceleration site coincides with or is adjacent to the magnetic reconnection site. The presence of open magnetic field lines is also supported by lowerfrequency radio observations including WIND detection of type III bursts indicating escaping energetic electrons.

To complete the 3D modeling we populated the flaring loop (i.e., the region of the closed field lines shown by green with the central field line shown by red) with appropriate nonuniform spatial distributions of the

\footnotetext{
8 WWW.lmsal.com/solarsoft/ssw_packages_info.html
}
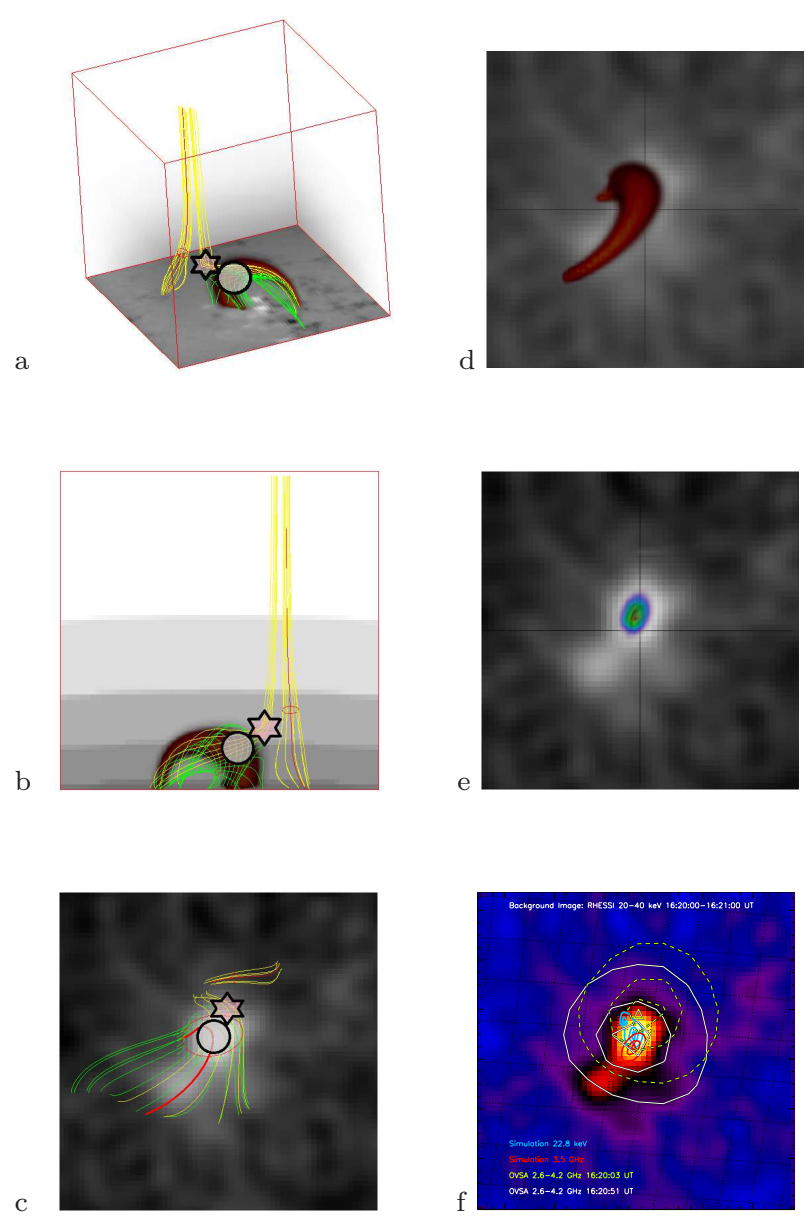

FIG. 10. 3D model of the flaring region based on LFFF $\left(\alpha \approx-5.5 \cdot 10^{-10} \mathrm{~cm}^{-1}\right.$ ) extrapolation of the photospheric SOHO/MDI magnetogram. (a-c) Extrapolated 3D magnetic data cube visualized by two magnetic flux tubes (central field lines are red): one consists mainly of closed field lines (green) with a few outer open field lines (yellow), while the other one consists of open field lines only. The locations of the acceleration region (star symbol) and trapping source (circle) are shown. (a) perspective view with the line-of-sight magnetogram shown at the bottom boundary; the dark red structure represents the model spatial distribution of the thermal plasma. (b) side view of the structure; step-like grey stripes represent the assumed hydrostatic plasma distribution in the corona outside the flaring loop. (c) top view of the structure (thermal distribution not shown); the greyscale background shows the HXR image taken at 16:20:00-16:21:00 UT. (d) top view of the thermal plasma distribution, $(e)$ nonthermal electron distribution, and $(f)$ a combination of simulated and observed images. The same HXR image is used as a background in all these panels. The contours at the right bottom panel are for $30 \%$, $70 \%$, and $90 \%$ of the corresponding peak value. Centroid of the acceleration region (the OVSA image at 16:20:03 UT), which defines the location of the star symbols in $(a-c)$, is separately shown by the green triangle.

thermal plasma (red in panels $a$ and $d$ ) and nonthermal electrons (rainbow colors in panel $e$ ). We then use this 3D model to compute the corresponding radio and $\mathrm{X}$-ray images by solving the corresponding radiation transfer equations numerically. Results of this modeling in the form of simulated images at $22.8 \mathrm{keV}$ and $3.5 \mathrm{GHz}$ are overplotted on top of the observed radio and $\mathrm{X}$-ray images. One can clearly see that the locations of the simulated images are consistent with each other and with observed locations of the HXR source and late-stage (trapped) radio source, while 
the simulated radio source from the trapped component is clearly displaced compared with radio location of the acceleration region.

The global parameters of the acceleration region are $B \sim 120 \mathrm{G}$ and $V \sim 6 \cdot 10^{27} \mathrm{~cm}^{-3}$, which are, respectively, two times and ten times larger than reported in the earlier cold, tenuous (2002 July 30) flare (Fleishman et al. 2011). The residence time of the fast electrons at the acceleration region is $\sim 3 \mathrm{~s}$, which is comparable to that in the cold, tenuous flare, and is much longer than the free-streaming time through the acceleration region. As noted in Fleishman et al. (2011), this favors diffusive electron transport due to their scattering by turbulent waves and, thus, a stochastic acceleration mechanism. The available data is, unfortunately, insufficient to firmly specify the version of stochastic acceleration mechanism (see e.g. Petrosian 2012, for a recent review) operating in the event; however, it does favor those models predicting a roughly energy-independent diffusion time at the source, like in the cold flare event (Fleishman et al. 2011)

Properties of the accelerated electron components are somewhat different from those in the 2002 July 30 cold flare. Firstly, in the 2002 April 11 event the accelerated electron spectrum is noticeably softer $(\delta \approx 5)$ than in the cold flare $(\delta \approx 3.5)$. Secondly, in the cold flare the accelerated electrons are detected at the energies above $6 \mathrm{keV}$, while in the April 11 event they are only seen above $\sim 20 \mathrm{keV}$; lower-energy $\mathrm{X}$-ray emission is dominated by the thermal background. Thirdly, the acceleration efficiency is different: in the cold flare almost all available electrons were accelerated, while in the April 11 event even the peak instantaneous number density of the fast electrons $\left(n_{r} \sim 2 \cdot 10^{8} \mathrm{~cm}^{-3}\right)$ does not exceed $10 \%$ of the thermal electron density. Fourthly, in the April 11 event we clearly see a spectral evolution indicative of the growth of a power-law tail ( $E_{\max }$ increases with time at the acceleration stage), whereas no spectral evolution was detected in the cold flare event, which implies a nearly instantaneous growth of the powerlaw tail. And finally, the released flare energy divides between the thermal and nonthermal components in remarkably different proportions in these two events.

Let us discuss from whence all these differences could originate. We have already concluded that the bulk acceleration mechanism is likely to be a stochastic/Fermi process with a relatively long residence time of the electrons controlled by their spatial diffusion on the turbulent magnetic irregularities at the acceleration region. For a diffusive Fermi acceleration process the shape of the particle energy spectrum depends primarily on the ratio of two key parameters - the acceleration rate $\tau_{a}$ (this is the time needed to establish the nonthermal particle spectrum, not to be interpreted as a duration of the acceleration process) and the residence/diffusion time of the electrons $\tau_{d}$ at the acceleration region in such a way that the larger the $\tau_{a} / \tau_{d}$ ratio the steeper (softer) the accelerated electron spectrum, (see, e.g., Hamilton \& Petrosian 1992). The residence times, $\tau_{d} \sim 3 \mathrm{~s}$, are comparable in the two events under comparison; the acceleration rates are, however, different. Indeed, the acceleration time $\tau_{a}$ can be roughly estimated as the time needed for the power-law tail to grow, which is clearly shorter than the residence time, $\tau_{a}<3 \mathrm{~s}$, in the cold flare (recall, no spectral evolution was noted), while longer, $\tau_{a}>3 \mathrm{~s}$, in the April 11 event ( $E_{\max }$ increases with time). Thus, for other conditions being equal, the accelerated electron energy spectral index must be larger in the April 11 event in agreement with observations.

The acceleration efficiency and energy balance in the flare depend, in addition to the acceleration mechanism itself, on the process of electron extraction from the thermal pool and their injection into the main acceleration process. In the cold flare almost all available thermal electrons were injected and accelerated, although their consequent energy losses were insufficient to significantly heat the thermal plasma. In contrast, in the April 11 event, only a relatively minor fraction of the thermal electrons were accelerated, making the collisional heating of the thermal plasma even less efficient than in the cold flare case (given that other relevant physical parameters are similar in these two cases). Thus, the presence of a very hot flaring SXR plasma with $T \sim 20 \mathrm{MK}$ (which is present even before the flare impulsive phase) requires another heating mechanism distinct from the collisional plasma heating by accelerated electrons. This conclusion is further supported by the spatial displacement between the thermal SXR source and nonthermal coronal HXR and microwave sources.

Although the available data are insufficient to firmly identify the flare energization process in either event, or the mechanism of energy division between the thermal and nonthermal components, we can conclude that this process does show some resemblance to that controlling the balance between Joule heating and runaway electrons in a DC electric field. Indeed, suppose that there is a relatively weak sub-Dreicer electric field directed along the flaring loop magnetic field. This electric field will initiate an electric current, which will lose its energy through Joule heating, while the fraction of the runaway electrons available for further stochastic acceleration will be relatively minor. In the case of a stronger electric field, e.g., comparable to the Dreicer field, the fraction of the runaway electrons becomes large, while the Joule heating is reduced so the plasma heating is modest. Even though it is a long way from these speculations to even a qualitative model, the analysis performed favors a flare picture in which electrons are first extracted from the thermal pool by a DC electric field (of yet unspecified origin) and then stochastically accelerated to form a power-law-like energy distribution. Therefore, a stochastic acceleration mechanism naturally containing a DC electric field is called for.

We have shown that observations of radio emission directly from the acceleration site provide important constraints on the acceleration mechanism in solar flares. Despite the great differences between this flare and the cold flare, a similar acceleration mechanism, although operating in a somewhat different parameter regime, seems to be called for. Future radio spectral imaging observations that can better separate the acceleration site from the sites of trapping and precipi- 
tation are needed to investigate the flare acceleration mechanism(s) in more detail.

This work was supported in part by NSF grants AGS-0961867, AST-0908344, AGS-1250374 and NASA grants NNX10AF27G and NNX11AB49G to New Jersey Institute of Technology and by the RFBR grants 12-02-00173 and 12-02-00616. This work was supported by a UK STFC rolling grant and the Leverhulme Trust, UK. This work also benefited from workshop support from the International Space Science Institute (ISSI). Financial support by the European Commission through HESPE (FP7-SPACE2010-263086) (EPK) and the "Radiosun" (PEOPLE2011-IRSES-295272) Networks is gratefully acknowledged.

\section{REFERENCES}

Altyntsev, A. T., Fleishman, G. D., Huang, G.-L., \& Melnikov, V. F. 2008, ApJ, 677, 1367

Aschwanden, M. J. 1998, ApJ, 502, 455

Bastian, T. S. 2006, in Solar Physics with the Nobeyama Radioheliograph, 3-10

Bastian, T. S., Benz, A. O., \& Gary, D. E. 1998, ARA\&A, 36, 131

Bastian, T. S., Fleishman, G. D., \& Gary, D. E. 2007, ApJ, 666,1256

Battaglia, M., \& Kontar, E. P. 2011, ApJ, 735, 42

Bespalov, P. A., Zaitsev, V. V., \& Stepanov, A. V. 1987, Sol. Phys., 114, 127

Emslie, A. G., Kontar, E. P., Krucker, S., \& Lin, R. P. 2003, ApJ, 595, L107

Fleishman, G. D., Kontar, E. P., Nita, G. M., \& Gary, D. E. 2011, ApJ, 731, L19

Fleishman, G. D., \& Kuznetsov, A. A. 2010, ApJ, 721, 1127

Fleishman, G. D., \& Melnikov, V. F. 2003, ApJ, 584, 1071

Fleishman, G. D., Nita, G. M., \& Gary, D. E. 2009, ApJ, 698, L183

Hamilton, R. J., \& Petrosian, V. 1992, ApJ, 398, 350

Haug, E. 1997, A\&A, 326, 417

Holman, G. D. 2012, ApJ, 745, 52

Kontar, E. P., Hannah, I. G., Jeffrey, N. L. S., \& Battaglia, M. 2010, ApJ, 717, 250

Kontar, E. P., MacKinnon, A. L., Schwartz, R. A., \& Brown, J. C. 2006, A\&A, 446, 1157

Kosugi, T., et al. 1992, PASJ, 44, L45

Kundu, M. R., White, S. M., Shibasaki, K., Sakurai, T., \& Grechnev, V. V. 2001, ApJ, 547, 1090
Kuznetsov, A. A., Nita, G. M., \& Fleishman, G. D. 2011, ArXiv e-prints

Lee, J., \& Gary, D. E. 2000, ApJ, 543, 457

Li, Y., \& Fleishman, G. D. 2009, ApJ, 701, L52

Lin, R. P., et al. 2002, Sol. Phys., 210, 3

Melnikov, V. F. 1994, Radiophysics and Quantum Electronics, 37, 557

Melnikov, V. F. 2006, in Solar Physics with the Nobeyama Radioheliograph, 11-22

Melnikov, V. F., \& Magun, A. 1998, Sol. Phys., 178, 153

Melnikov, V. F., Shibasaki, K., \& Reznikova, V. E. 2002 ApJ, 580, L185

Melrose, D. B., \& Brown, J. C. 1976, MNRAS, 176, 15

Messmer, P., Benz, A. O., \& Monstein, C. 1999, Sol. Phys., 187,335

Nita, G. M., Gary, D. E., \& Lee, J. 2004, ApJ, 605, 528

Park, S., \& Fleishman, G. D. 2010, Sol. Phys., 266, 323

Petrosian, V. 2012, Space Sci. Rev., 49

Qiu, J., Gary, D. E., \& Fleishman, G. D. 2009, Sol. Phys., 255, 107

Reznikova, V. E., Melnikov, V. F., Shibasaki, K., Gorbikov, S. P., Pyatakov, N. P., Myagkova, I. N., \& Ji, H. 2009, ApJ, 697,735

Schwartz, R. A., Csillaghy, A., Tolbert, A. K., Hurford, G. J., Mc Tiernan, J., \& Zarro, D. 2002, Sol. Phys., 210, 165

Stepanov, A. V., \& Tsap, Y. T. 2002, Sol. Phys., 211, 135

Su, Y., Holman, G. D., \& Dennis, B. R. 2011, ApJ, 731, 106

Tzatzakis, V., Nindos, A., \& Alissandrakis, C. E. 2008, Sol. Phys., 253, 79

van den Oord, G. H. J. 1990, A\&A, 234, 496

Xu, Y., Emslie, A. G., \& Hurford, G. J. 2008, ApJ, 673, 576 

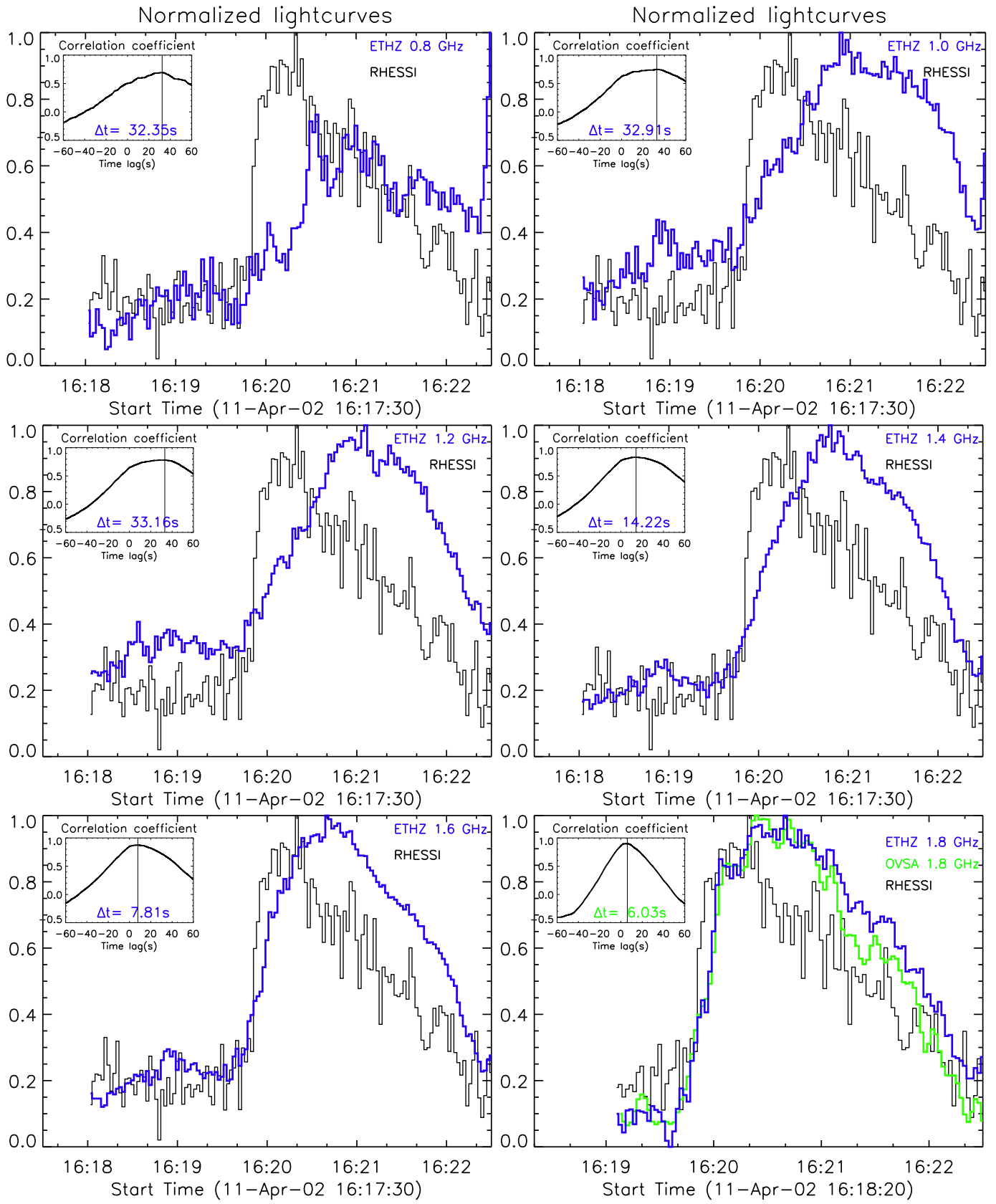

FIg. 11.- Radio to HXR timing with Phoenix-2 and OVSA at different frequencies. Black thin curve, same throughout all the panels: HXR (20-40 keV) light curve with $2 \mathrm{~s}$ resolution normalized to 1; blue thick curves: resampled (to the RHESSI $2 \mathrm{~s}$ resolution) Phoenix-2 light curves at different frequencies indicated at the panels; green curves: spline interpolated OVSA light curves from original $4 \mathrm{~s}$ resolution to the RHESSI $2 \mathrm{~s}$ resolution at different frequencies indicated at the panels. The insets: corresponding Radio-to-HXR lag correlations in which the time delay is printed by either blue (for Phoenix-2 data) or green (for OVSA data). 

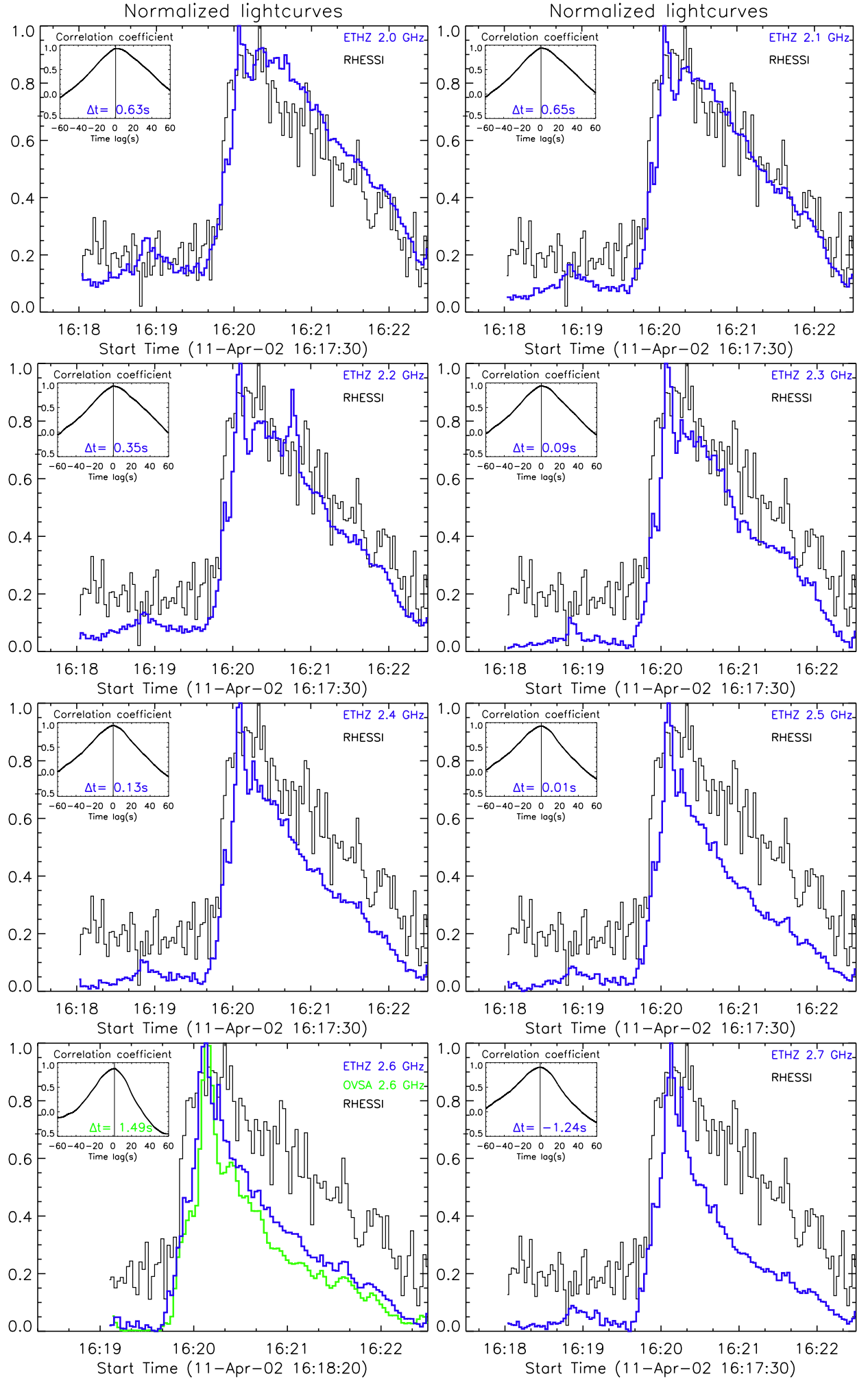

FIG. 12.- Radio to HXR timing; continued. 

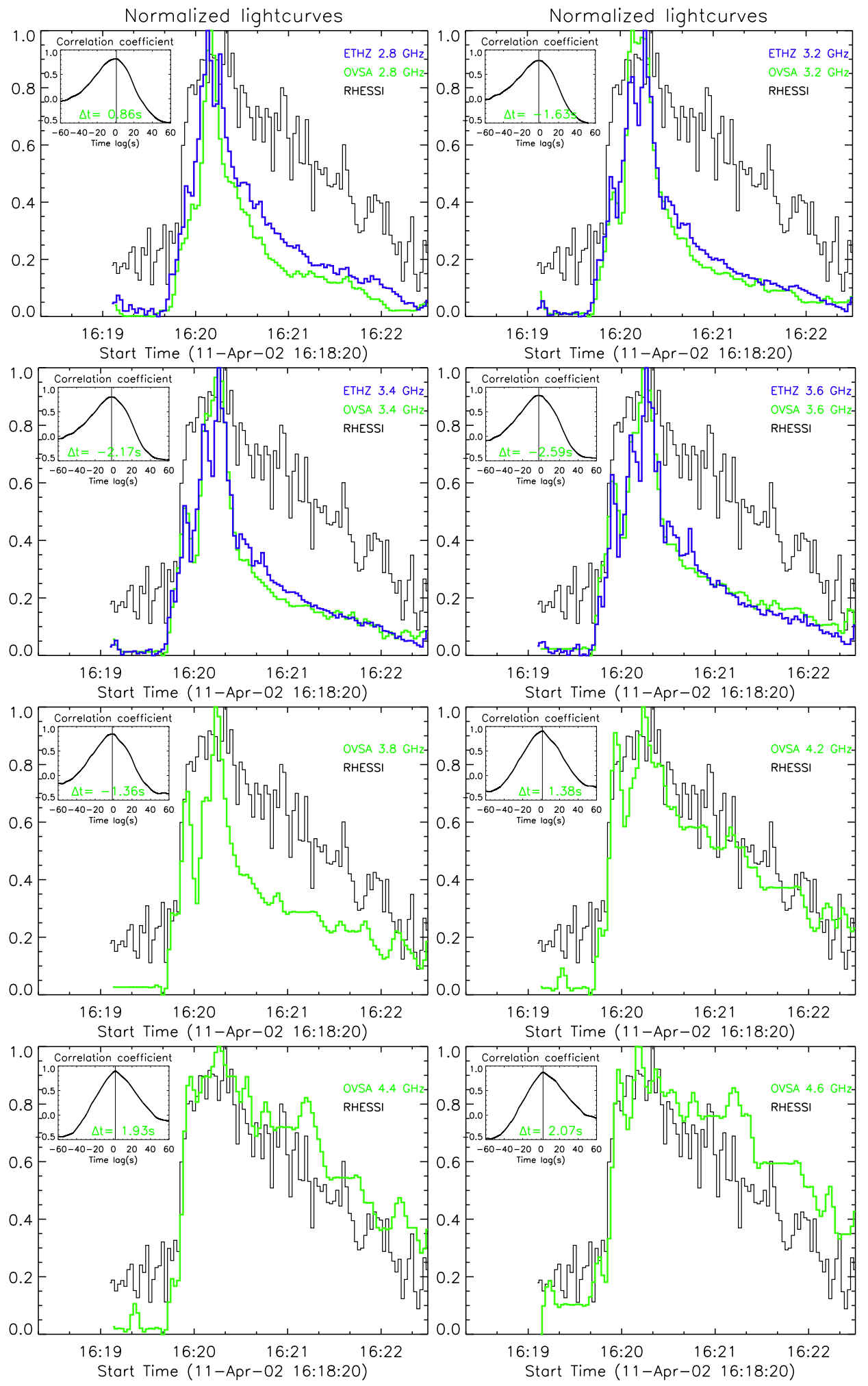

FIG. 13.- Radio to HXR timing; continued. 

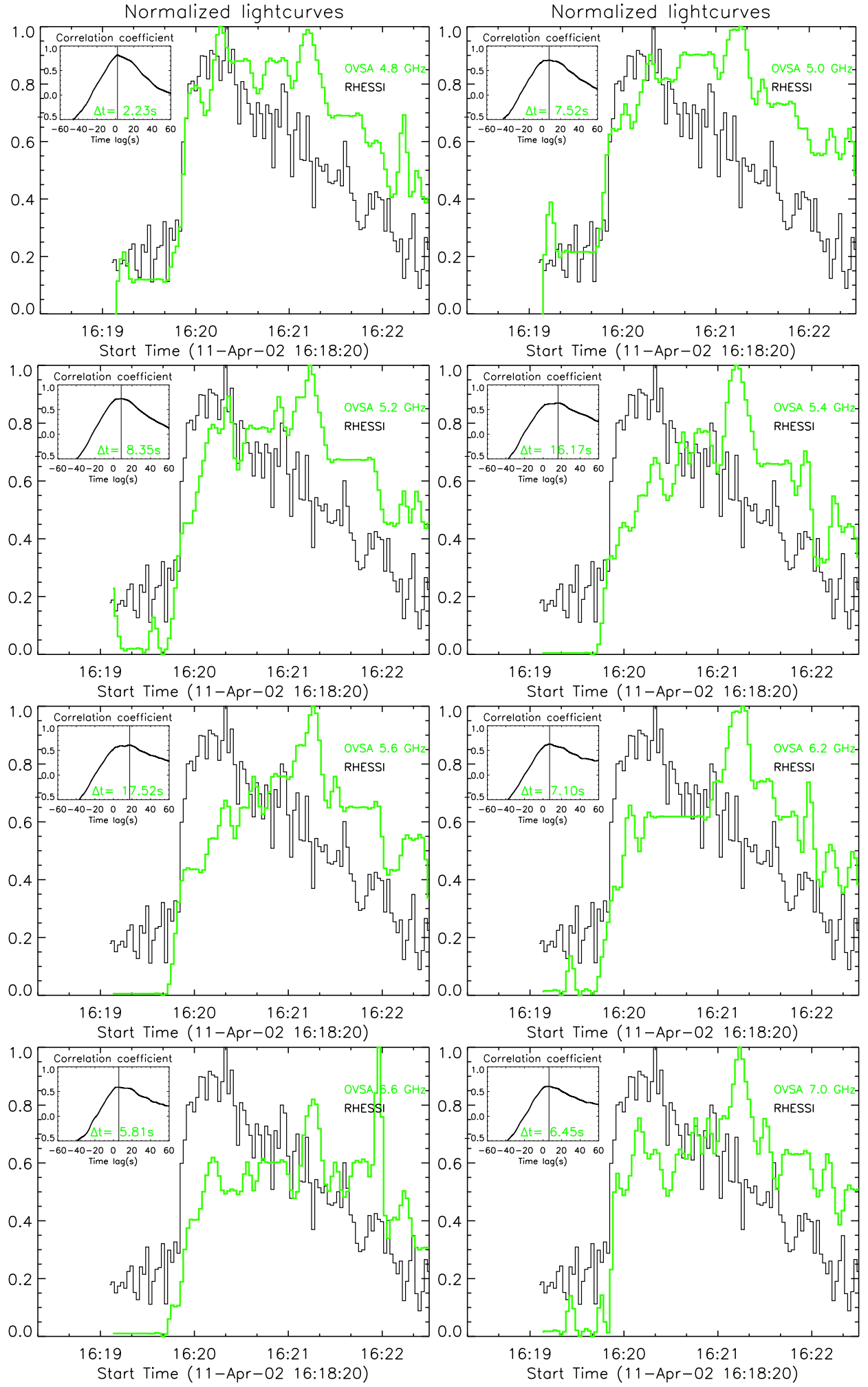

FIG. 14.- Radio to HXR timing; continued. 


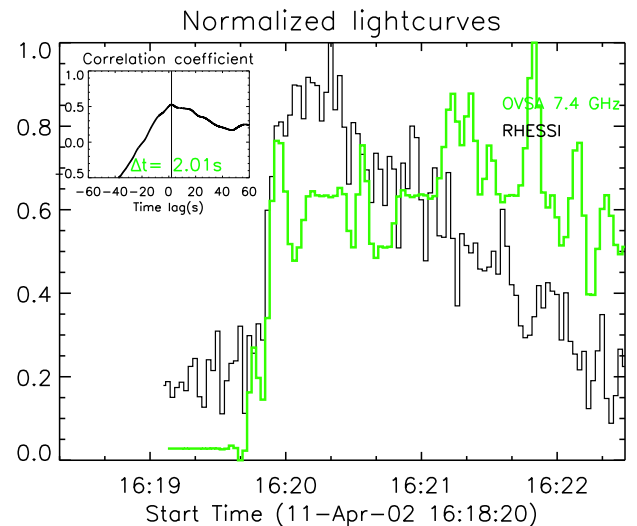

Normalized lightcurves
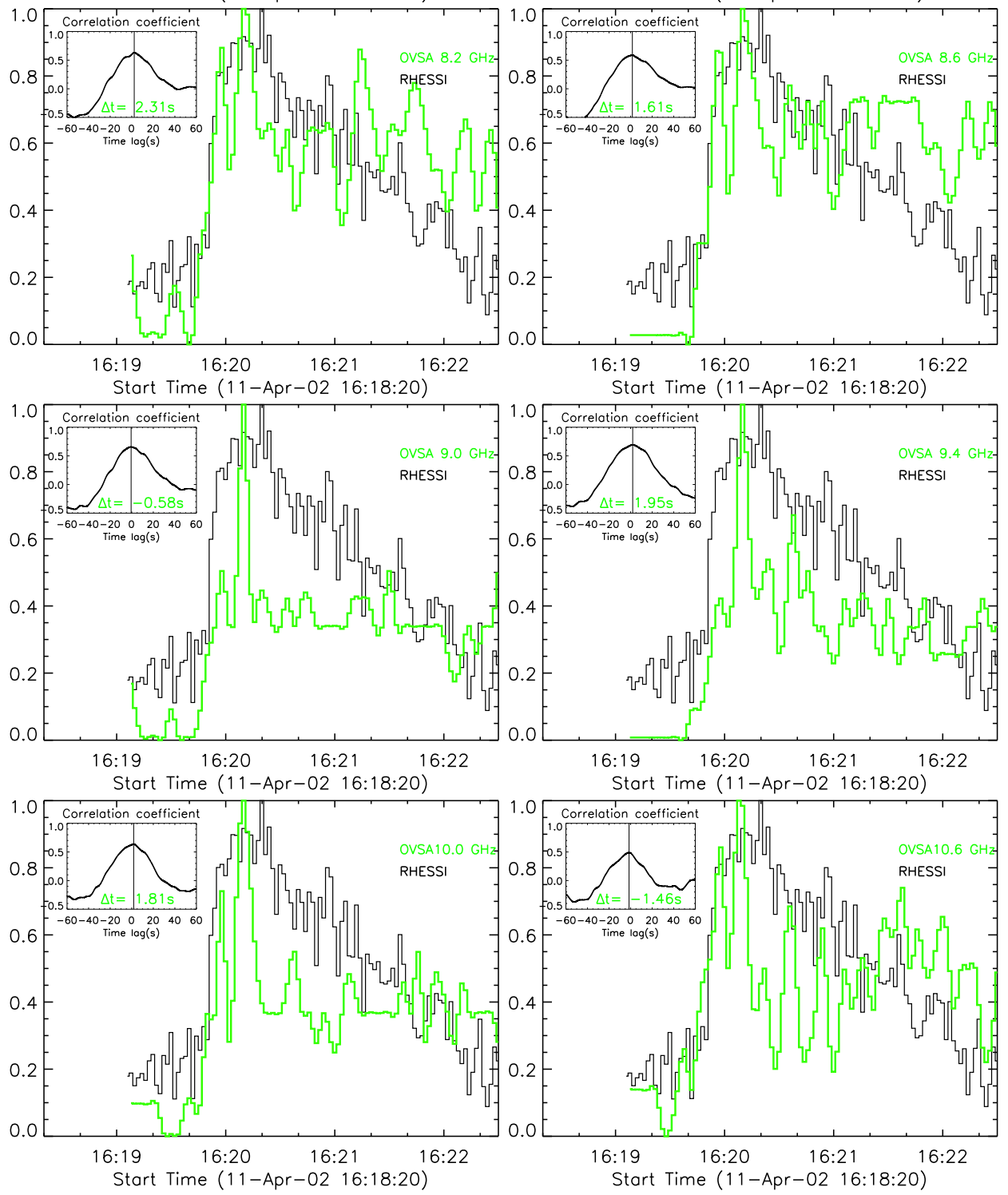

FIG. 15.- Radio to HXR timing; continued. 


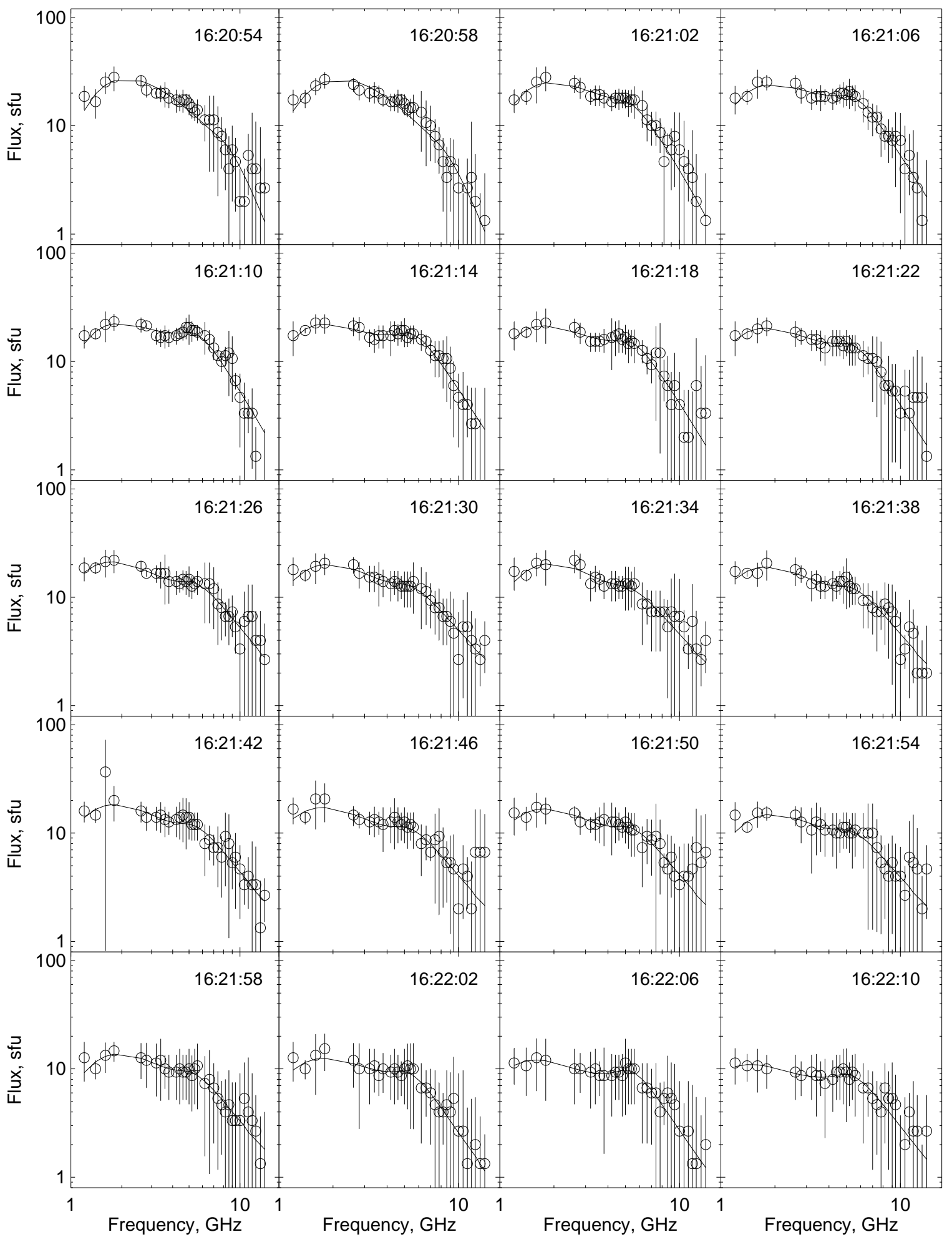

FIG. 16. - Radio spectral fit of the OVSA spectra; Part II. 


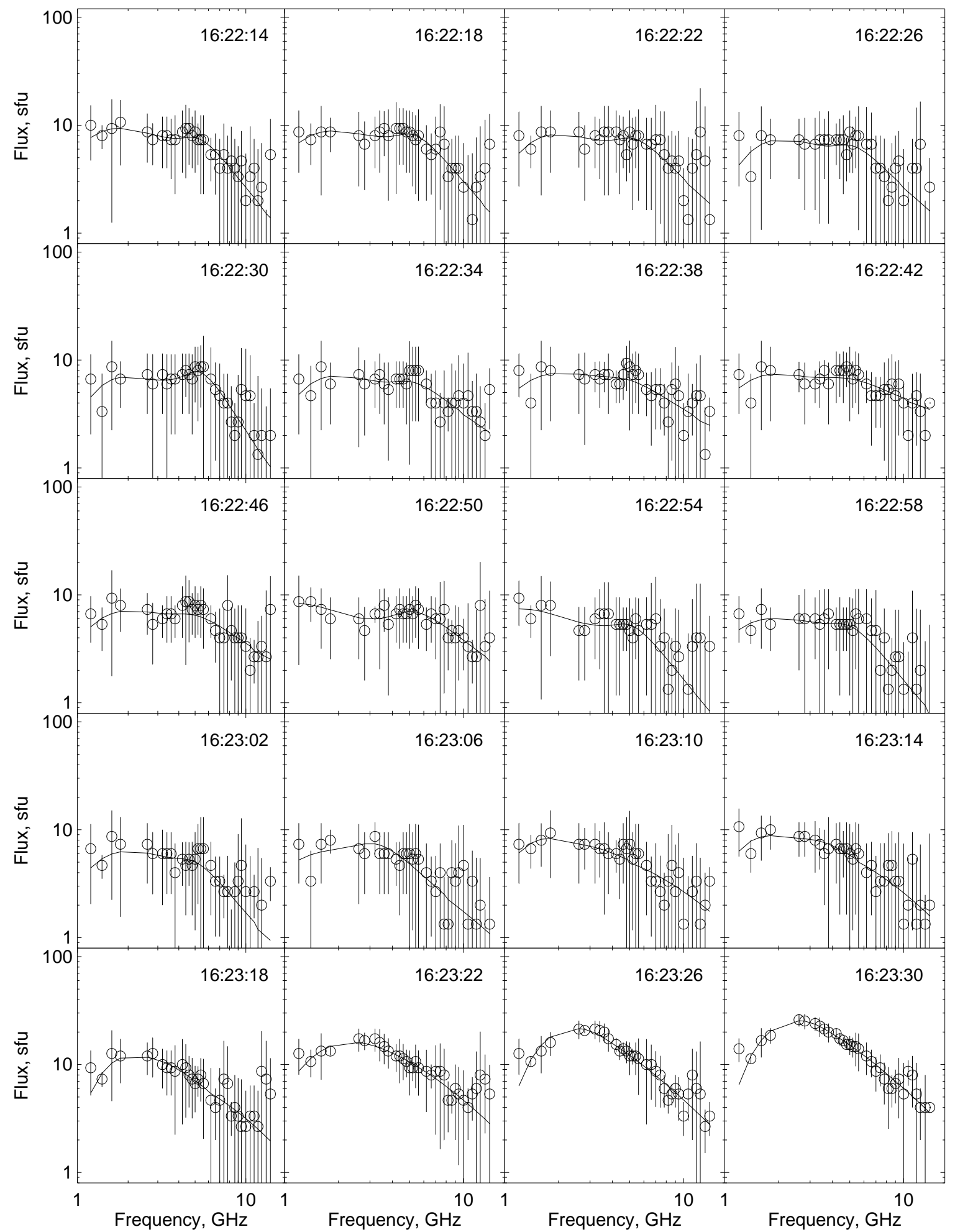

FIG. 17.- Radio spectral fit of the OVSA spectra; Part III. 


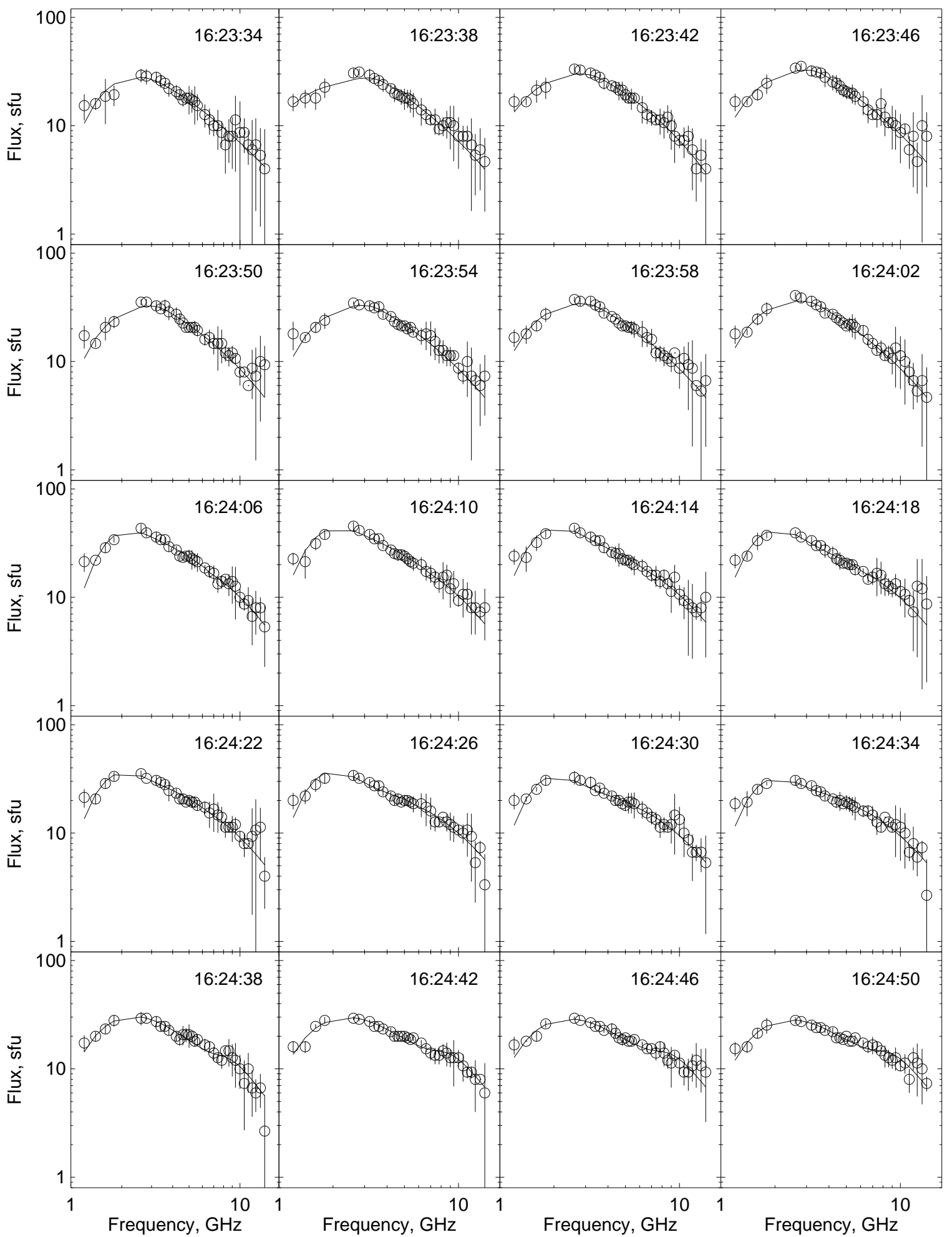

FIG. 18. - Radio spectral fit of the OVSA spectra; Part IV. 


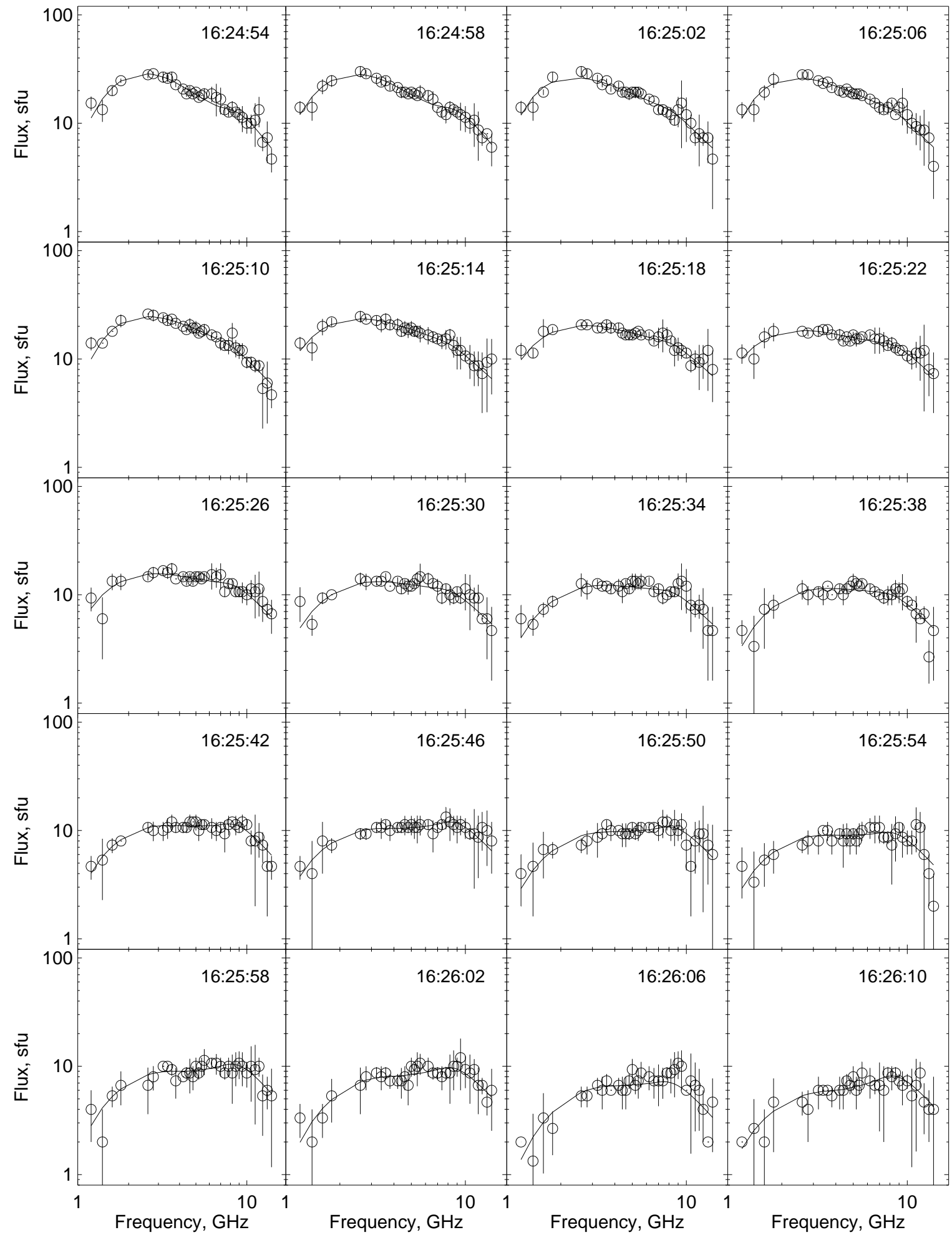

FIG. 19. - Radio spectral fit of the OVSA spectra; Part V. 